\title{
The component fairness solution for cycle-free graph games
}

Citation for published version (APA):

Herings, P. J. J., van der Laan, G., \& Talman, A. J. J. (2005). The component fairness solution for cyclefree graph games. METEOR, Maastricht University School of Business and Economics. METEOR Research Memorandum No. 057 https://doi.org/10.26481/umamet.2005057

Document status and date:

Published: 01/01/2005

DOI:

10.26481/umamet.2005057

Document Version:

Publisher's PDF, also known as Version of record

\section{Please check the document version of this publication:}

- A submitted manuscript is the version of the article upon submission and before peer-review. There can be important differences between the submitted version and the official published version of record.

People interested in the research are advised to contact the author for the final version of the publication, or visit the DOI to the publisher's website.

- The final author version and the galley proof are versions of the publication after peer review.

- The final published version features the final layout of the paper including the volume, issue and page numbers.

Link to publication

\footnotetext{
General rights rights.

- You may freely distribute the URL identifying the publication in the public portal. please follow below link for the End User Agreement:

www.umlib.nl/taverne-license

Take down policy

If you believe that this document breaches copyright please contact us at:

repository@maastrichtuniversity.nl

providing details and we will investigate your claim.
}

Copyright and moral rights for the publications made accessible in the public portal are retained by the authors and/or other copyright owners and it is a condition of accessing publications that users recognise and abide by the legal requirements associated with these

- Users may download and print one copy of any publication from the public portal for the purpose of private study or research.

- You may not further distribute the material or use it for any profit-making activity or commercial gain

If the publication is distributed under the terms of Article $25 \mathrm{fa}$ of the Dutch Copyright Act, indicated by the "Taverne" license above, 


\title{
The Component Fairness Solution for Cycle-Free Graph Games
}

\author{
P. Jean-Jacques Herings* Gerard van der Laan ${ }^{\dagger}$ Dolf Talman ${ }^{\ddagger}$
}

December 15, 2005

*P.J.J. Herings, Department of Economics, Maastricht University, P.O. Box 616, 6200 MD Maastricht, The Netherlands, e-mail: P.Herings@algec.unimaas.nl.

${ }^{\dagger}$ G. van der Laan, Department of Econometrics and Tinbergen Institute, Vrije Universiteit, De Boelelaan 1105, 1081 HV Amsterdam, The Netherlands, e-mail: glaan@feweb.vu.nl.

$\ddagger$ A.J.J. Talman, Department of Econometrics \& Operations Research and CentER, Tilburg University, P.O. Box 90153, 5000 LE Tilburg, The Netherlands, e-mail:talman@uvt.nl. 


\begin{abstract}
In this paper we study cooperative games with limited cooperation possibilities, represented by an undirected cycle-free communication graph. Players in the game can cooperate if and only if they are connected in the graph, i.e. they can communicate with one another. We introduce a new single-valued solution concept, the component fairness solution. Our solution is characterized by component efficiency and component fairness. The interpretation of component fairness is that deleting a link between two players yields for both resulting components the same average change in payoff, where the average is taken over the players in the component. Component fairness replaces the axiom of fairness characterizing the Myerson value, where the players whose link is deleted face the same loss in payoff. The component fairness solution is always in the core of the restricted game in case the game is superadditive and can be easily computed as the average of $n$ specific marginal vectors, where $n$ is the number of players. We also show that the component fairness solution can be generated by a specific distribution of the Harsanyi-dividends.
\end{abstract}

Keywords: TU-game, communication structure, Myerson value, fairness, marginal vector.

AMS subject classification: 90B18, 91A12, 91A43.

JEL code: C71. 


\section{Introduction}

A situation in which a finite set of players can obtain certain payoffs by cooperation can be described by a cooperative game with transferable utility, or simply a TU-game, being a pair $(N, v)$, where $N$ is a finite set of players and $v: 2^{N} \rightarrow \mathbb{R}$ is a characteristic function on $N$ such that $v(\emptyset)=0$. For any subset $S \in 2^{N}, v(S)$ is the worth of coalition $S$, i.e. the members of coalition $S$ can obtain a total payoff of $v(S)$ by agreeing to cooperate. Unless stated otherwise, we assume that $N=\{1, \ldots, n\}$, i.e. $N$ is a set of $n$ players, indexed by $i=1, \ldots, n$, and we denote a game $(N, v)$ shortly by its characteristic function $v$.

A payoff vector $x \in \mathbb{R}^{n}$ of a game $v$ is an $n$-dimensional vector giving a payoff $x_{i} \in \mathbb{R}$ to any player $i \in N$. In the following we denote $x(S)=\sum_{i \in S} x_{i}, S \in 2^{N}$. A payoff vector $x$ is efficient if it exactly distributes the worth $v(N)$ of the 'grand coalition' $N$, i.e. if $x(N)=v(N)$. A solution for TU-games is a mapping $F$ that assigns to every game $v$ a set of payoff vectors $F(v) \subset \mathbb{R}^{n}$. A solution $F$ is efficient if for any game $v$ every element in $F(v)$ is efficient. A classical solution is the core, see Gillies [6], which assigns to any game $v$ the set $C(v)$ of undominated efficient payoff vectors, i.e. $x(N)=v(N)$ and $x(S) \geq v(S)$ for all $S \in 2^{N}$. The best known single-valued solution is the Shapley value, see Shapley [15], assigning to any game $v$ the average $\psi(v)$ of all marginal vectors. For some permutation $\pi$ on $N$, the corresponding marginal vector $m^{\pi}(v) \in \mathbb{R}^{n}$ assigns to every player $i$ a payoff $m_{i}^{\pi}(v)=v\left(\pi^{i} \cup\{i\}\right)-v\left(\pi^{i}\right)$, where $\pi^{i}=\{j \in N \mid \pi(j)<\pi(i)\}$, i.e. $\pi^{i}$ is the set of players preceding $i$ in the permutation $\pi$. Since every marginal vector is efficient, the Shapley value is an efficient solution. A game $v$ is convex if $v(S \cup T)+v(S \cap T) \geq v(S)+v(T)$ for all $S, T \in 2^{N}$ and superadditive if $v(S \cup T) \geq v(S)+v(T)$ for all $S, T \in 2^{N}$ such that $S \cap T=\emptyset$. The core of a game is equal to the convex hull of all marginal vectors if and only if the game is convex. In that case the Shapley value is an element of the core. In general, the Shapley value may be outside the core, even if the core is not empty. Moreover, the core is nonempty if and only if the game is balanced, see Bondareva [1].

In this paper we study cooperative games with limited cooperation possibilities, represented by an undirected communication graph as introduced by Myerson [12]. The vertices in the graph represent the players and the edges represent the communication links between the players. Players can only cooperate if they are connected. This yields a socalled graph game, given by a triple $(N, v, L)$ with $N$ the set of players, $v$ the characteristic function and $L$ the set of edges (communication links) in the graph $(N, L)$ on the set of nodes $N$. Since in graph games only connected coalitions can cooperate, the core reduces to the set of component efficient payoff vectors that are not dominated by any connected coalition, i.e.

$$
C(v, L)=\left\{x \in \mathbb{R}^{n} \mid x(S)=v(S), S \in C_{m}^{L}(N), \text { and } x(S) \geq v(S), S \in C^{L}(N)\right\},
$$


where $C^{L}(N)$ is the collection of all connected subsets of $N$ in the graph $(N, L)$ and $C_{m}^{L}(N)$ is the collection of all components of $N$ in the graph, see also Section 2 for these notions. The set $C(v, L)$ equals the core $C\left(v^{L}\right)$ of the so-called restricted game $v^{L}$, defined by Myerson [12] as

$$
v^{L}(S)=\sum_{T \in C_{m}^{L}(S)} v(T), S \in 2^{N}
$$

where $C_{m}^{L}(S)$ is the collection of components of $S$ induced by the graph $(N, L)$. A singlevalued solution for graph games is a function $f$ that assigns to every graph game $(N, v, L)$ a unique payoff vector $f(v, L) \in \mathbb{R}^{n}$. The so-called Myerson value, to be denoted by $\mu$, is the solution that assigns to any graph game the Shapley value of the restricted game, so $\mu(v, L)=\psi\left(v^{L}\right)$. The Myerson value can be characterized by component efficiency, i.e. $\sum_{i \in S} \mu_{i}(v, L)=v(S)$ if $S \in C_{m}^{L}(N)$, and fairness. The latter property says that deleting a link between two players yields for both players the same change in payoff. Clearly, the Myerson value is the average of all marginal vectors of $v^{L}$ and is guaranteed to be in the core if the restricted game is convex. Alternative characterizations of the Myerson value have been given in Myerson [13] and Borm et al. [2]. In the latter paper also another solution for graph games has been proposed, the so-called positional value, see also Meessen [11]. This value is also characterized by component efficiency and by balanced total threats, see Slikker [16]. The balanced total threat property says that the total threat of any player towards another player equals to the total threat of that player towards the first player, where the total threat of a player towards another player is the sum over all links of the first player of payoff differences the second player experiences if such a link is broken.

From Kaneko and Wooders [9] and Le Breton, Owen and Weber [10] it follows that $C\left(v^{L}\right)$ is not empty if the graph $(N, L)$ contains no cycles and $v$ is superadditive, see also Demange [4]. For an arbitrarily taken node, a cycle-free graph induces a tree with the given node as root. Demange assigns to any node taken as root a particular marginal vector and shows that this vector is in the core of the restricted game $v^{L}$. Interpreting the tree as a hierarchy on the set of players, Demange [5] argues that hierarchies yield stability, in the sense that there exist undominated payoff vectors under the mild condition of superadditivity, providing a rationale for the fact that a group organizes itself in a hierarchy to achieve coordination.

In this paper we provide a new single-valued solution concept for cycle-free graph games. To do so we replace Myerson's fairness by an alternative fairness property, to be called component fairness. This property says that deleting a link between two players yields for both resulting components the same average change in payoff, where the average is taken over the players in the component. The new solution is characterized by component efficiency and component fairness. It is easy to compute the new solution. We prove that 
it equals the average of the $n$ marginal vectors considered by Demange. It follows that the component fairness solution belongs to the core of the restricted game if the game is superadditive. So, contrary to the Myerson value and the position value the new value is in that case always an element of the core. We also compare the three values in terms of distributions of the so-called Harsanyi-dividends, see Harsanyi [8].

This paper has been organized as follows. In Section 2 we give some preliminary notions of graph theory. In Section 3 we introduce the concept of component fairness solution and prove that component efficiency and component fairness fully characterize the component fairness solution on the class of cycle-free graph games. We also show that it equals the average of $n$ specific marginal vectors, for each player one. In Section 4 we show that in case the game is superadditive the component fairness solution lies in the core and we compare the new value with the Myerson value and the position value. Section 5 concludes.

\section{Some Notions in Graph Theory}

In this section we present some notions in graph theory that are needed for our results. An undirected graph is a pair $(N, L)$ where $N$ is a set of nodes ${ }^{1}$ and $L$ is a collection of edges, i.e. $L \subseteq\{\{i, j\} \mid i, j \in N, i \neq j\}$ is a collection of subsets of $N$ such that each element of $L$ contains precisely two elements of $N$. Node $j$ is adjacent to node $i$ if $\{i, j\} \in L$. Because the elements of $L$ will represent binary communication links between the players, in the sequel we call them links instead of edges. For $K \in 2^{N}$, the graph $(K, L(K))$ with $L(K)=\{l \in L \mid l \subseteq K\}$ is called the subgraph of $(N, L)$ on $K$. The number of nodes in $K$ being adjacent to node $i \in K$ in subgraph $(K, L(K))$ is called $i$ 's degree in $(K, L(K))$ and is given by $d_{K}^{L}(i)=|\{j \in K \mid\{i, j\} \in L(K)\}|$.

A sequence of $k$ different nodes $\left(i_{1}, \ldots, i_{k}\right)$ is a path in a graph $(N, L)$ if $\left\{i_{h}, i_{h+1}\right\} \in L$ for $h=1, \ldots, k-1$. Two nodes $i, j \in N$ are connected in the graph $(N, L)$ if there exists a path $\left(i_{1}, \ldots, i_{k}\right)$ with $i_{1}=i$ and $i_{k}=j$. A graph $(N, L)$ is connected if any two nodes $i, j \in N$ are connected in $(N, L)$. In a given graph $(N, L)$, a set of nodes $K$ is said to be a connected subset of $N$ when the subgraph $(K, L(K))$ is connected. A subset $K$ of $N$ is a component of $(N, L)$ if the subgraph $(K, L(K))$ is maximally connected, i.e. $(K, L(K))$ is connected and for any $j \in N \backslash K$, the subgraph $(K \cup\{j\}, L(K \cup\{j\}))$ is not connected. Note that the collection of components of $(N, L)$ forms a partition of $N$. The collection of all connected subsets of $K$ in the subgraph $(K, L(K))$ of $(N, L)$ is denoted by $C^{L}(K)$. The collection of all components of $(N, L)$ is denoted by $C_{m}^{L}(N)$. A sequence of nodes

\footnotetext{
${ }^{1}$ Since in this paper the nodes in a graph represent the players in a game we use the same notation for both the set of nodes and the set of players.
} 
$\left(i_{1}, \ldots, i_{k+1}\right)$ is a cycle in $(N, L)$ if (i) $k \geq 2$, (ii) all nodes $i_{1}, \ldots, i_{k}$ are different elements of $N$, (iii) $i_{k+1}=i_{1}$ and (iv) $\left\{i_{h}, i_{h+1}\right\} \in L$ for $h=1, \ldots, k$. A graph $(N, L)$ is cycle-free when it does not contain any cycle.

A directed graph is a pair $(N, D)$ where $N$ is a set of nodes and $D$ is a collection of directed edges, i.e. $D \subseteq\{(i, j) \mid i, j \in N, i \neq j\}$. If $(i, j) \in D$, then the node $j$ is a successor of $i$ and $i$ is a predecessor of $j$. We say that $j \neq i$ is a subordinate of $i$ if there is a sequence of directed edges $\left(i_{h}, i_{h+1}\right) \in D, h=1, \ldots, k$, such that $i_{1}=i$ and $i_{k+1}=j$. We denote the set of subordinates of $i$ in $(N, D)$ by $S_{D}(i)$ and denote $S_{D}^{c}(i)=S_{D}(i) \cup\{i\}$. For $K \in 2^{N}$, the directed graph $(K, D(K))$ with $D(K)=\{(i, j) \in D \mid i, j \in K\}$ is called the directed subgraph of $(N, L)$ on $K$. A directed graph $(N, D)$ is a tree if there is one node in $N$, called the root or top-node, having no predecessors in $D$ and there is a unique sequence of directed edges in $(N, D)$ from this node to any other node in $N$. Let $(N, \bar{D})$ be the undirected graph induced by $(N, D)$, i.e. $\bar{D}=\{\{i, j\} \mid(i, j) \in D\}$. A directed $\operatorname{graph}(N, D)$ is a forest if for every component $K$ of $(N, \bar{D})$ the directed subgraph $(K, D(K))$ is a tree. A directed graph $(N, D)$ represents a hierarchy on $N$ when $(N, D)$ is a forest. Clearly, in a forest $(N, D)$ we have that $S_{D}^{c}(j) \subseteq S_{D}(i)$ when $j$ is a subordinate of $i$.

Finally, let $(N, L)$ be a cycle-free undirected graph and $K$ a component of $(N, L)$ consisting of $k=|K|$ nodes. Then every node $i \in K$ induces a unique tree $T(i)$ on $K$ in the following way. For any $j \in K \backslash\{i\}$, take the unique path in $(K, L(K))$ from $i$ to $j$, then change the undirected edges on this path to directed edges in such a way that the first node in any ordered pair is the node that comes first on the path from $i$ to $j$. In this way the undirected cycle-free subgraph $(K, L(K))$ induces $k$ different trees, one tree for each of the $k$ different nodes of $K$. For a component $K$ of $(N, L)$, we denote by $T_{L}^{K}$ the collection of all $|K|$ trees on $K$ induced by the undirected graph $(N, L)$.

\section{Component Fairness Solution}

In this section we define a new solution concept for cycle-free graph games and we prove that this is the unique solution satisfying the properties of component efficiency and component fairness. Let $(N, v, L)$ be a cycle-free graph game. For a component $K$ of $(N, L)$ and $i \in K$, let $T(i) \in T_{L}^{K}$ be the unique tree with player $i$ as its top-player and, with a slight abuse of notation, let $S_{T(i)}(j)$ and $S_{T(i)}^{c}(j)$ be the sets of subordinates of $j$ in $T(i)$, without and including $j$, respectively. Further, define by $L_{T(i)}(j)=\{h \in K \mid(j, h) \in T(i)\}$ the set of successors of $j$ in $T(i)$. We now associate to player $j$ in $T(i)$ payoffs $t_{j}^{i}(v, L)$ given by

$$
t_{j}^{i}(v, L)=v\left(S_{T(i)}^{c}(j)\right)-\sum_{h \in L_{T(i)}(j)} v\left(S_{T(i)}^{c}(h)\right) .
$$


So, the payoff to player $j \in K$ is equal to the worth of the coalition consisting of player $j$ and all his subordinates in $T(i)$ minus the sum of the worths of the coalitions consisting of any successor of player $j$ and all subordinates of this successor in $T(i)$. By the tree structure of $T(i)$ we have that for every player $j \in K$ the sets $S_{T(i)}^{c}(h), h \in L_{T(i)}(j)$, form a partitioning of $S_{T(i)}(j)$ and thus

$$
t_{j}^{i}(v, L)=v\left(S_{T(i)}^{c}(j)\right)-v^{L}\left(S_{T(i)}(j)\right),
$$

where the second right hand term is the restricted worth of the coalition consisting of all subordinates of $j$ in the tree $T(i)$. So, the payoff to player $j$ in tree $T(i)$ is equal to what player $j$ contributes when he joins his subordinates in $T(i)$. Clearly, the set $S_{T(i)}^{c}(j)$ itself is connected, so when joining his subordinates, player $j$ connects all the subsets of subordinates of his successors to one connected set and receives his marginal contribution to it. Observe that a player $j \in K$ receives his own worth $v(\{j\})$ when $j$ has no subordinates in the tree $T(i)$. More generally, the total payoff to a player $j$ and all his subordinates in $T(i)$ is equal to the worth of the coalition $S_{T(i)}^{c}(j)$, i.e.

$$
\sum_{h \in S_{T(i)}^{c}(j)} t_{h}^{i}(v, L)=v\left(S_{T(i)}^{c}(j)\right), \quad j \in K .
$$

We now define the Component Fairness solution $C F$ as the solution assigning to each cyclefree graph game $(N, v, L)$ the payoff vector in which player $j$ in a component $K \in C_{m}^{L}(N)$ receives the average of all the payoffs $t_{j}^{i}(v, L)$ in the $|K|$ trees $T(i), i \in K$, in $T_{L}^{K}$.

\section{Definition 3.1 Component Fairness Solution}

On the class of cycle-free graph games, the Component Fairness (CF) solution assigns to any $(N, v, L)$ the payoff vector $C F(v, L)$ given by

$$
C F_{j}(v, L)=\frac{1}{|K|} \sum_{i \in K} t_{j}^{i}(v, L), \quad j \in K, \quad K \in C_{m}^{L}(N) .
$$

To characterize the CF-solution we state the following properties for a solution $f$ on the class of cycle-free graph games.

\section{Axiom 3.2 Component Efficiency}

A solution $f$ on the class of cycle-free graph games is component efficient if for any game $(N, v, L)$ it holds that $\sum_{i \in K} f_{i}(v, L)=v(K)$ for each $K \in C_{m}^{L}(N)$.

To state the next axiom, for a component $K$ and a link $\{i, j\} \in L(K)$, let $K^{h}$ be the component in $(N, L \backslash\{i, j\})$ containing $h, h=i, j$. Clearly, $K^{h}$ is the subset of $K$ of players connected to $h$ in the graph that results after deleting the link $\{i, j\}$. 


\section{Axiom 3.3 Component Fairness}

A solution $f$ on the class of cycle-free graph games satisfies component fairness if for any $(N, v, L)$ it holds that

$$
\frac{1}{\left|K^{i}\right|} \sum_{h \in K^{i}}\left(f_{h}(v, L)-f_{h}(v, L \backslash\{i, j\})\right)=\frac{1}{\left|K^{j}\right|} \sum_{h \in K^{j}}\left(f_{h}(v, L)-f_{h}(v, L \backslash\{i, j\})\right),
$$

for all $\{i, j\} \in L(K), K \in C_{m}^{L}(N)$.

The first property is standard and has been introduced by Myerson [12]. The second property is an alternative to the Myerson fairness property which states that $f_{i}(v, L)-$ $f_{i}(v, L \backslash\{i, j\})=f_{j}(v, L)-f_{j}(v, L \backslash\{i, j\})$ for any $\{i, j\} \in L$. The Myerson fairness property states that two players $i$ and $j$ linked directly together face the same loss in payoff when the link between them is deleted from the set of links. On the class of cycle-free graphs, any link is crucial because deleting a link breaks the component into two parts. Considering a link $\{i, j\}$ in a component $K$ we have that all players in $K^{i}$ are only connected to the players in $K^{j}$ through the link between $i$ and $j$. One may argue that $h, h=i, j$, behaves on behalf of all players in $K^{h}$ in sustaining the link $\{i, j\}$. The component fairness property states then that when a link $\{i, j\}$ between $i$ and $j$ is deleted, the average change in the payoffs of the players in $K^{i}$ is equal to the average change in payoffs of the players in $K^{j}$. Observe that superadditivity and component efficiency implies that on average the players face a loss in payoff when a link is deleted.

We now state the main theorem, which says that the CF-solution is the unique solution satisfying component efficiency and component fairness.

Theorem 3.4 A solution $f$ on the class of cycle-free graph games is equal to the CFsolution if and only if it satisfies component efficiency and component fairness.

\section{Proof.}

First, suppose that $f$ satisfies component efficiency and component fairness. For any graph game $(N, v, L)$, let $l=|L|$ be the number of links and $c=\left|C_{m}^{L}(N)\right|$ the number of components in $(N, L)$. Observe that $l+c=n$, because the graph is cycle-free. Component efficiency implies that

$$
\sum_{h \in K} f_{h}(v, L)=v(K), \text { for all } K \in C_{m}^{L}(N) .
$$

Also because of component efficiency we have $\sum_{h \in K^{i}} f_{h}(v, L \backslash\{i, j\})=v\left(K^{i}\right)$ and $\sum_{h \in K^{j}}$ $f_{h}(v, L \backslash\{i, j\})=v\left(K^{j}\right)$ for any link $\{i, j\}$ in $L(K)$. Therefore, component fairness reduces to

$$
\frac{1}{\left|K^{i}\right|}\left(\sum_{h \in K^{i}} f_{h}(v, L)-v\left(K^{i}\right)\right)=\frac{1}{\left|K^{j}\right|}\left(\sum_{h \in K^{j}} f_{h}(v, L)-v\left(K^{j}\right)\right),
$$


for all $\{i, j\} \in L(K)$ and $K \in C_{m}^{L}(N){ }^{2}$ Since there are $c$ equations of type (3.4) and $l$ equations of type (3.5) and all the $c+l=n$ equations are linearly independent, these equations uniquely determine $f(v, L)$.

It remains to prove that the CF-solution satisfies the two properties. First, since $i \in K$ is the top-player in the tree $T(i)$ on component $K$ and thus $S_{T(i)}^{c}(i)=K$, for any $i \in K$ it follows from equation (3.3) that

$$
\sum_{h \in K} t_{h}^{i}(v, L)=\sum_{h \in S_{T(i)}^{c}(i)} t_{h}^{i}(v, L)=v\left(S_{T(i)}^{c}(i)\right)=v(K) .
$$

Hence,

$$
\begin{aligned}
\sum_{h \in K} \mathrm{CF}_{h}(v, L) & =\sum_{h \in K} \frac{1}{|K|} \sum_{i \in K} t_{h}^{i}(v, L) \\
& =\frac{1}{|K|} \sum_{i \in K} \sum_{h \in K} t_{h}^{i}(v, L)=\frac{1}{|K|} \sum_{i \in K} v(K)=v(K),
\end{aligned}
$$

which shows component efficiency.

To show component fairness, suppose link $\{i, j\}$ is deleted in some component $K$. Component efficiency implies that for both components $K^{i}$ and $K^{j}$ in the graph $(N, L \backslash$ $\{i, j\})$ it holds that $\sum_{h^{\prime} \in K^{h}} \mathrm{CF}_{h^{\prime}}(v, L \backslash\{i, j\})=v\left(K^{h}\right), h=i, j$. Further, consider the tree $T(h)$ on $K$ for some $h \in K^{i}$, then by definition of $t^{h}(v, L)$ we have that

$$
\sum_{h^{\prime} \in K^{j}} t_{h^{\prime}}^{h}(v, L)=v\left(K^{j}\right)
$$

On the other hand, for $h \in K^{j}$ it holds that

$$
\sum_{h^{\prime} \in K^{j}} t_{h^{\prime}}^{h}(v, L)=v(K)-v\left(K^{i}\right)
$$

Since there are $\left|K^{i}\right|$ equations of type (3.6) and $\left|K^{j}\right|$ equations of type (3.7), we obtain that

$$
\sum_{h \in K^{j}} \mathrm{CF}_{h}(v, L)=\frac{1}{|K|}\left(\left|K^{i}\right| v\left(K^{j}\right)+\left|K^{j}\right|\left(v(K)-v\left(K^{i}\right)\right)\right) .
$$

With $\left|K^{i}\right|+\left|K^{j}\right|=|K|$ it follows that

$$
\begin{aligned}
\sum_{h \in K^{j}}\left(\mathrm{CF}_{h}(v, L)-\mathrm{CF}_{h}(v, L \backslash\{i, j\})\right) & = \\
\frac{1}{|K|}\left(\left|K^{i}\right| v\left(K^{j}\right)+\left|K^{j}\right|\left(v(K)-v\left(K^{i}\right)\right)\right)-v\left(K^{j}\right) & =\frac{\left|K^{j}\right|}{|K|}\left(v(K)-v\left(K^{i}\right)-v\left(K^{j}\right)\right) .
\end{aligned}
$$

\footnotetext{
${ }^{2}$ Observe that because of this reduced form of the component fairness property, it is not needed to use induction on the number of links, as in the proof of the uniqueness of the Myerson value, in which one first has to determine the values $f_{h}(v, L \backslash\{i, j\})$ for $h=i, j$.
} 
Analogously it follows that

$$
\sum_{h \in K^{i}}\left(\mathrm{CF}_{h}(v, L)-\mathrm{CF}_{h}(v, L \backslash\{i, j\})\right)=\frac{\left|K^{i}\right|}{|K|}\left(v(K)-v\left(K^{i}\right)-v\left(K^{j}\right)\right),
$$

which shows that $\mathrm{CF}$ satisfies component fairness.

From the proof it follows that the CF-solution satisfies the property that when a link $\{i, j\}$ connects two subcomponents $K^{i}$ and $K^{j}$ to form component $K$ the surplus $v(K)-$ $v\left(K^{i}\right)-v\left(K^{j}\right)$ obtained from this connection is distributed among the two subcomponents proportional to the number of players in these subcomponents. In the next section we will consider some more properties of the CF-solution and compare this solution with other solutions for graph games.

\section{Properties and Comparison}

Consider a connected cycle-free graph $(N, L)$, so that $N$ is the unique component. Then the CF-solution becomes

$$
\mathrm{CF}_{j}(v, L)=\frac{1}{n} \sum_{i \in N} t_{j}^{i}(v, L), \quad j \in N
$$

where $t^{i}(v, L) \in \mathbb{R}^{n}$ is the payoff vector defined by equation (3.1) when $i$ is the top-player in the tree $T(i)$ on $N$ induced by $(N, L)$. Suppose that $v$ is superadditive. It has been shown by Demange [5] that the vector $t^{i}(v, L)$ in which a player $j$ gets a payoff equal to the worth of the coalition consisting of himself with all his subordinates minus the restricted worth of the coalition of his subordinates is in the core of the restricted game $v^{L}$. In fact, it holds that $v^{L}$ is permutationally convex for any permutation $\pi$ satisfying $\pi_{j}<\pi_{h}$ when $j$ is a subordinate of $h$ on a tree $T(i)$ on $N$, see for instance van Velzen [17]. According to Granot and Huberman [7] this implies that the corresponding marginal vector $m^{\pi}$ is in the core of $v^{L}$. It is straightforward to verify that $t^{i}(v, L)=m^{\pi}\left(v^{L}\right)$ for any permutation $\pi$ that satisfies the condition above for the tree $T(i)$. Since the core is convex and $\operatorname{CF}(v, L)$ is the average of all vectors $t^{i}(v, L), i \in N$, this implies that the CF-solution is stable in the sense that it is in the core of $v^{L}$ and thus cannot be dominated by any coalition. The discussion above generalizes straigtforwardly in case the graph $(N, L)$ consists of several components. Since stability implies the weaker condition of component efficiency, we have the following important corollary.

\section{Corollary 4.1}

On the class of cycle-free superadditive graph games the CF-solution is the unique solution satisfying stability and component fairness. 
The corollary not only says that the CF-solution is the unique stable solution satisfying component fairness, it also says that there exists a solution being stable and component fair. This makes the CF-solution an attractive alternative to other solutions such as the Myerson value and the position value which may not be stable, even not on the class of cycle-free games. The Myerson value $\mu(v, L)$, characterized by component efficiency and fairness, satisfies, for any link $\{i, j\} \in L$,

$$
\mu_{i}(v, L)-\mu_{i}(v, L \backslash\{i, j\})=\mu_{j}(v, L)-\mu_{j}(v, L \backslash\{i, j\}) .
$$

It is equal to the Shapley value $\psi\left(v^{L}\right)$ of the restricted game $v^{L}$, implying that it may be outside the core of $v^{L}$ when $v^{L}$ is not convex. So, fairness may contradict the requirement of stability. Also the position value, denoted by $\gamma(v, L)$, introduced for the class of all graph games and characterized on the class of cycle-free graph games in Borm et al. [2], does not need to be stable. Slikker [16] characterizes the position value on the class of graph games as the unique solution satisfying component efficiency and balanced total threats, saying that the total threat of any player $i$ towards another player $j$ is equal to the total threat of player $j$ towards player $i$. The total threat of a player $i$ towards another player $j$ is defined as the sum over all links of player $i$ of the payoff differences player $j$ experiences if such a link is broken, i.e. for each pair of players $i, j \in N$ it holds that

$$
\sum_{h \mid\{i, h\} \in L}\left(\gamma_{j}(v, L)-\gamma_{j}(v, L \backslash\{i, h\})\right)=\sum_{h \mid\{j, h\} \in L}\left(\gamma_{i}(v, L)-\gamma_{i}(v, L \backslash\{j, h\})\right) .
$$

The next example shows that also the balanced threat property may contradict the requirement of stability, i.e. also the position value may be outside the core.

Example 4.2 Let $(N, v, L)$ be given by $N=\{1,2,3\}, v(\{1,2\})=1, v(\{2,3\})=v(N)=2$, $v(S)=0$, otherwise and $L=\{\{1,2\},\{2,3\}\}$. Observe that $v^{L}=v$, so that the Myerson value equals the Shapley value: $\mu(v, L)=\psi\left(v^{L}\right)=\psi(v)=(1 / 6,7 / 6,2 / 3)^{\top}$. To obtain the position value, straightforward calculations show that $\gamma(v, L \backslash\{1,2\})=(0,1,1)^{\top}$ and $\gamma(v, L \backslash\{2,3\})=\left(\frac{1}{2}, \frac{1}{2}, 0\right)^{\top}$. Then, it follows that $\gamma(v, L)=\left(\frac{1}{4}, 1, \frac{3}{4}\right)^{\top}$ by solving the system of three equations given by the component efficiency property and the two balanced threat property equations between 1 and 2 and between 3 and 2. Since $x_{1}=0$ for any payoff vector $x$ in the core of $v^{L}$, this shows that both the Myerson value and the position value are not stable. Finally, the component fairness solution follows easily as the average of the three vectors $t^{i}(v, L), i=1,2,3$, and is given by $\mathrm{CF}(v, L)=(0,5 / 3,1 / 3)$, which is in the core.

Next we compare the three values in terms of distributions of the so-called Harsanyi dividends. Let $\Omega$ be the collection of nonempty subsets of $N$. Then, for $T \in \Omega$, the unanimity game $u_{T}$ on $N$ is given by $u_{T}(S)=1$ if $T \subseteq S$, and $u_{T}(S)=0$ otherwise. 
Leaving out $v(\emptyset)$, it is well-kown that the collection of unanimity games forms a basis in $\mathbb{R}^{2^{n}-1}$ for the class of TU-games on $N$, i.e. for any game $v$ represented as a $\left(2^{n}-1\right)$-vector with the worths of the non-empty coalitions as its components, it holds that

$$
v=\sum_{S \in \Omega} \Delta^{S}(v) u_{S}
$$

where the coefficients $\Delta^{S}(v)$ are the Harsanyi dividends, see Harsanyi [8], given by

$$
\Delta^{S}(v)=\sum_{T \subseteq S}(-1)^{|S|-|T|} v(T), \quad S \in \Omega .
$$

It is well-known that the Shapley value can be obtained by distributing the Harsanyi dividend of coalition $S$ equivalently among the players in $S$,

$$
\psi_{i}(v)=\sum_{S \in \Omega \mid i \in S} \frac{1}{|S|} \Delta^{S}(v), \quad i \in N .
$$

The Shapley payoff to player $i$ is equal to the sum over all coalitions $S$ containing player $i$ of a share $\frac{1}{|S|}$ of the dividend of coalition $S$.

Consider a cycle-free graph game $(N, v, L)$. For a connected set $S$, a node $i \in S$ is extreme if $S \backslash\{i\}$ is connected in the subgraph $(N \backslash\{i\}, L(N \backslash\{i\}))$. The set of extreme nodes of $S$ is denoted by $\operatorname{Ex}(S)$. Owen [14], see also Borm et al. [2], showed that the dividends of the restricted game can be expressed as sum of the dividends of the underlying game $v$,

$$
\Delta^{S}\left(v^{L}\right)= \begin{cases}\sum_{T \in \Sigma(S)} \Delta^{T}(v), & \text { if } S \text { is connected } \\ 0, & \text { otherwise }\end{cases}
$$

where $\Sigma(S)=\{T \subseteq S \mid E x(S) \subseteq T\}$. Since the dividends of not connected coalitions are zero, for the restricted game of a cycle-free graph game $(N, v, L)$ equation (4.8) reduces to

$$
v^{L}=\sum_{S \in C^{L}(N)} \Delta^{S}\left(v^{L}\right) u_{S}
$$

and the payoffs according to the Myerson value follow by distributing the dividends of the connected coalitions equally among its players

$$
\mu_{i}(v, L)=\sum_{S \in C^{L}(N) \mid i \in S} \frac{1}{|S|} \Delta^{S}\left(v^{L}\right), i \in N .
$$

As follows from Borm et al. [2], see also van den Brink et al. [3], the position value is obtained by distributing the dividend of any connected coalition $S$ proportional among its players according to the degrees of the players in the subgraph $(S, L(S))$, i.e. the payoff of player $i$ according to the position value is given by

$$
\gamma_{i}(v, L)=\sum_{S \in C^{L}(N) \mid i \in S} \frac{d_{S}^{L}(i)}{\sum_{j \in S} d_{S}^{L}(j)} \Delta^{S}\left(v^{L}\right), i \in N .
$$


Comparing the two values shows that in the position value the more central players, that means players with more neighbours, get higher shares in the dividends. ${ }^{3}$

Also the component fairness solution can be obtained by distributing the dividends appropriately. To derive these distributions, for each connected $S$ in $(N, L)$ and $j \in S$, let $p_{S}^{L}(j)$ be the number of players outside $S$ represented by $j$ in $S$. We say that player $j \in S$ represents player $k$ outside $S$, if $k$ is connected to $j$ and on the unique path connecting $j$ and $k$ all players between $j$ and $k$ are outside $S$. So,

$$
p_{S}^{L}(j)=\sum_{h \in N \backslash S \mid\{j, h\} \in L}\left|K^{h}\right|,
$$

where, as in the previous section, $K^{h}$ is the component of the graph $(N, L \backslash\{j, h\})$ containing player $h$. Clearly, player $j$ connects the players in $S$ with the players in $K^{h}$ in the sense that link $\{j, h\}$ is on the path between any player $i$ in $S$ and any player $k$ in the set $K^{h} \subseteq N \backslash S$. Notice that for some players $j, p_{S}^{L}(j)=0$. We now have the following result.

Theorem 4.3 The payoff of player $i$ according to the CF-solution is given by

$$
C F_{i}(v, L)=\sum_{S \in C^{L}(N) \mid i \in S} \frac{1+p_{S}^{L}(i)}{|S|+\sum_{j \in S} p_{S}^{L}(j)} \Delta^{S}\left(v^{L}\right), i \in N
$$

\section{Proof.}

First, observe that $\mathrm{CF}$ is linear, i.e. for two games $v$ and $w$ and coefficients $\alpha, \beta \in \mathbb{R}$, it holds that $\mathrm{CF}(\alpha v+\beta w, L)=\alpha \mathrm{CF}(v, L)+\beta \mathrm{CF}(w, L)$. This follows from the fact that the $\mathrm{CF}$-solution is the average of specific marginal vectors of the restricted game $v^{L}$, where the choice of the marginal vectors is independent of the payoffs. Further, observe that $u_{S}^{L}=u_{S}$ if $S$ is connected in $(N, L)$. Hence, with equation (4.10) it follows that

$$
\mathrm{CF}_{i}(v, L)=\sum_{S \in C^{L}(N)} \mathrm{CF}_{i}\left(u_{S}, L\right) \Delta^{S}\left(v^{L}\right), i \in N .
$$

Let $S$ be a connected coalition in $(N, L)$ and let $T \in C_{m}^{L}(N)$ be the component of $(N, L)$ containing $S$. Since $u_{S}(K)=0$ for each component $K \neq T$, it follows from component efficiency and component fairness that $\mathrm{CF}_{j}\left(u_{S}, L\right)=0$ for all $j \notin T$. Further $u_{S}(T)=1$, so component efficiency requires that $\sum_{j \in T} \mathrm{CF}_{j}\left(u_{S}, L\right)=1$. Consider players $j \in T \backslash S$ and $i \in S$ and on the path $\left(i_{1}, \ldots, i_{r}\right)$, with $i_{1}=j$ and $i_{r}=i$, from $j$ to $i$, let, for some index $t, i_{t}$ be the last player outside $S$ (and thus $i_{t+1}$ the first player in $S$ ). Then delete

\footnotetext{
${ }^{3}$ Since dividends may be negative, this does not necessarily mean that in the position value the more central players get higher payoffs than in the Myerson value. In the example above, the coalition $\{1,2\}$ has dividend 1 , coalition $\{2,3\}$ has dividend 2 and the grand coalition $N$ has dividend -1 . Since in the latter coalition the central player 2 has degree two and the other players degree one, the position value gives a lower payoff to the central player 2 than the Myerson value.
} 
the link $\left\{i_{t}, i_{t+1}\right\}$ and consider the component $K^{i_{t}}$ in the graph $\left(N, L \backslash\left\{i_{t}, i_{t+1}\right\}\right)$. Then $j \in K^{i_{t}}$ and $S \subseteq K^{i_{t+1}}$. Since $u_{S}\left(K^{i_{t}}\right)=0$ and $u_{S}\left(K^{i_{t+1}}\right)=1$, component efficiency requires that $\sum_{h \in K^{i_{t}}} \mathrm{CF}_{h}\left(u_{S}, L \backslash\left\{i_{t}, i_{t+1}\right\}\right)=0$ and $\sum_{h \in K^{i_{t+1}}} \mathrm{CF}_{h}\left(u_{S}, L \backslash\left\{i_{t}, i_{t+1}\right\}\right)=1$. Since this holds for any $j \in T \backslash S$, it follows by applying the component fairness property that also $\mathrm{CF}_{j}\left(u_{S}, L\right)=0$ for all $j \in T \backslash S$ and thus for all $j$ not in $S$. Consequently, $\sum_{i \in S} \mathrm{CF}_{i}\left(u_{S}, L\right)=1$. Now, for any two linked players $i$ and $j$ in $S$, let $u_{S}^{L \backslash\{i, j\}}$ be the restricted game of $u_{S}$ on $(N, L \backslash\{i, j\})$. Since $S$ is not connected anymore, $u_{S}^{L \backslash\{i, j\}}(T)=0$ for any $T \in 2^{N}$, i.e. the restricted game is the null-game, yielding worth zero to any coalition. Consequently, $\mathrm{CF}_{j}\left(u_{S}, L \backslash\{i, j\}\right)=0$ for all $j$. Hence the component fairness property requires that for each pair $i, j \in S$ with $\{i, j\} \in L$,

$$
\sum_{h \in K^{i}} \mathrm{CF}_{h}\left(u_{S}, L\right)=\sum_{h \in K^{j}} \mathrm{CF}_{h}\left(u_{S}, L\right) .
$$

Since $S$ is connected, there are $|S|-1$ links in $S$ and thus $|S|-1$ of such equations. Together with $\sum_{i \in S} \mathrm{CF}_{i}\left(u_{S}, L\right)=1$ we have a system of $|S|$ linearly independent equations with $|S|$ unknowns, yielding a unique solution. It is easy to verify that the solution is given by

$$
\mathrm{CF}_{i}\left(u_{S}, L\right)=\frac{1+p_{S}^{L}(i)}{|S|+\sum_{j \in S} p_{S}^{L}(j)}, i \in S .
$$

Doing this for any connected $S$, the theorem follows from equation (4.10) and the linearity of the CF-solution.

Theorem 4.3 shows that the CF-solution can be obtained by distributing the dividend of any connected coalition $S$ among the players in $S$ in such a way that the share of player $i \in S$ in the dividend of coalition $S$ is given by $\left(1+p_{S}^{L}(i)\right) /\left(|S|+\sum_{j \in S} p_{S}^{L}(j)\right)$. The weight $1+p_{S}^{L}(i)$ is equal to the number of players on behalf of which player $i$ acts in $S$, including himself, i.e. one plus the number of players outside $S$ in the component containing $S$ and being connected to $i$ through a path of players outside $S$. Recall from above that the Myerson value is obtained by distributing the dividend of any connected coalition equally among the players in $S$ and the position value is obtained by distributing the dividends according to the degree of each player in the subgraph $(S, L(S))$. In both these cases the shares of the players in the dividends only depend on the structure of the subgraph $(S, L(S))$. In fact, the Myerson value only depends on the number of players in $S$. In contrast, for the CF-solution the shares also depend on the structure of the graph $(N, L)$ outside $S$. Indeed, the shares depend on the structure of the whole subgraph on the component $K$ containing $S$. As a result, the CF-solution for the unanimity game $u_{S}$ will change when the graph changes on $K$ but not on $S$, whereas the other two solutions 
can only change when the graph changes on $S$ itself. More precisely, when $\{i, j\}$ is a link of $L$ not on $S$ but on the component $K$ containing $S$, we have that

$$
\mu\left(u_{S}, L\right)=\mu\left(u_{S}, L \backslash\{i, j\}\right) \text { and } \gamma\left(u_{S}, L\right)=\gamma\left(u_{S}, L \backslash\{i, j\}\right),
$$

but

$$
\mathrm{CF}\left(u_{S}, L\right) \neq \mathrm{CF}\left(u_{S}, L \backslash\{i, j\}\right) .
$$

As has been shown in Borm et al. [2], both the Myerson value and the position value satisfy the so-called superfluous link property. A link $\{i, j\} \in L$ is superfluous in a graph game $(N, v, L)$ if $v^{E}(N)=v^{E \cup\{i, j\}}(N)$ for all $E \subseteq L \backslash\{i, j\}$, so when for each subset of links $E$ not containing $\{i, j\}$, the restricted value of the grand coalition on the graph $(N, E)$ is equal to the restricted value of it on the graph $(N, E \cup\{i, j\})$. A solution $f$ satisfies the superfluous link property if $f(v, L)=f(v, L \backslash\{i, j\})$ whenever $\{i, j\}$ is a superfluous link in $(v, L)$. Clearly, any link outside $S$ is a superfluous link in the graph game $\left(N, u_{S}, L\right)$. Since deleting such a link may change the CF-solution, CF does not satisfy the superfluous link property.

\section{Concluding Remarks}

In this paper we propose a new value for cycle-free graph games. In such games players are only able to cooperate if they are connected to each other. In case of superadditivity, the core of the game is non-empty and contains for each player a specific marginal vector. This marginal vector is induced by a tree in which that player is the root and the directed edges correspond to the undirected edges of the graph. The solution is then the average of these marginal vectors. Since all these marginal vectors are an element of the core, the new value is also an element of the core, even if the game is not convex. This is in contrast to the Myerson value and the position value. These values may not be elements of the core.

The new value has been axiomatized by component efficiency and component fairness. The latter property says that if a link is deleted, the average loss per player is the same in the two subcomponents resulting after deletion of the link. This means that the surplus in worth obtained from a link is distributed over these two subcomponents proportional to their number of players. In this respect the value differs from the Myerson value and the position value. The Myerson value is characterized by component efficiency and by fairness, the property that if a link is deleted, then the two players that were connected by this link have equal loss. This loss is therefore not related to the size of the components resulting after the deletion of the link. The position value is characterized by component efficiency and balanced total threats, the property that the total threat of a player towards 
another player is equal to the total threat of the latter player towards the former player. The component fairness solution may fail to satisfy balanced total threats.

In terms of distributions of Harsanyi-dividends the new solution is obtained by distributing the dividends of a connected coalition among its members in such a way that the share of a player in it is given by the relative number of players on which behalf the player acts in the coalition, including himself. This means that the shares depend on the structure of the subgraph on the component of which the coalition is a subset and not just on the structure of the subgraph on the coalition itself as is the case for the Myerson value and the position value. This property guarantees that the value is an element of the core.

It is not hard to compute the component fairness solution. If $n$ is the number of players, the value can be calculated as follows. First, for each player $i$, a permutation $\pi^{i}$ is determined satisfying that player $j$ has a higher rank than player $k$ if player $j$ lies on the unique path connecting player $i$ and player $k$. Any permutation that satisfies this condition leads to the same marginal vector. Next, the component fairness solution is the average of the $n$ marginal vectors obtained in this way. In general, the calculation of the Myerson value and the position value is more complex from a computational point of view. The Myerson value is the average of $n$ ! marginal vectors and the position value is the solution to a system of $n$ equations. For larger $n$ this may take quite some computational time. For example, if there are ten players, the new value is the average of just ten marginal vectors, whereas the Myerson value is the average of about 3.6 million marginal vectors and for the position value a system of ten equations has to be solved.

\section{References}

[1] Bondareva, O. (1963), Some Applications of Linear Programming Methods to the Theory of Cooperative Games, Problemy Kibernetiki, 10, 119-139 (in Russian).

[2] Borm, P., G. Owen, and S. Tijs (1992), On the Position Value for Communication Situations, SIAM Journal of Discrete Mathematics, 5, 305-320.

[3] Brink, R. van den, G. van der Laan, and V. Pruzhansky (2004), Harsanyi Power Solutions for Graph-Restricted Games, TI Discussion Paper 2004-095/1, Tinbergen Institute, Amsterdam/Rotterdam, The Netherlands.

[4] Demange, G. (1994), Intermediate Preferences and Stable Coalition Structures, Journal of Mathematical Economics, 23, 45-58.

[5] Demange, G. (2004), On Group Stability in Hierarchies and Networks, Journal of Political Economy, 112, 754-778. 
[6] Gillies, D.B. (1953), Some Theorems on n-Person Games, Princeton University Press, Princeton, NJ.

[7] Granot, G., and G. Huberman (1982), The Relationship Between Convex Games and Minimal Cost Spanning Tree Games: A Case for Permutationally Convex Games, SIAM Journal of Algebraic and Discrete Methods, 3, 288-292.

[8] Harsanyi, J.C. (1959), A Bargaining Model for Cooperative $n$-Person Games, in A.W. Tucker and R.D. Luce (eds.), Contributions to the Theory of Games IV, Princeton University Press, Princeton, NJ, pp. 325-355.

[9] Kaneko, M., and M.H. Wooders (1982), Cores of Partitioning Games, Mathematical Social Sciences, 3, 313-327.

[10] Le Breton, M., G. Owen, and S. Weber (1992), Strongly Balanced Cooperative Games, International Journal of Game Theory, 20, 419-427.

[11] Meessen, R. (1988), Communication Games, Master Thesis, University of Nijmegen, The Netherlands (in Dutch).

[12] Myerson, R.B. (1977), Graphs and Cooperation in Games, Mathematics of Operations Research, 2, 225-229.

[13] Myerson, R.B. (1980), Conference Structures and Fair Allocation Rules, International Journal of Game Theory, 9, 169-182.

[14] Owen, G. (1986), Values of Graph-Restricted Games, SIAM Journal on Algebraic and Discrete Methods, 7, 210-220.

[15] Shapley, L. (1953), A Value for n-Person Games, in H.W. Kuhn and A.W. Tucker (eds.), Contributions to the Theory of Games II, Princeton University Press, Princeton, NJ, pp. 307-317.

[16] Slikker, M. (2003), A Characterization of the Position Value, Beta Working Paper WP-96, TU Eindhoven, The Netherlands, to appear in International Journal of Game Theory.

[17] Velzen, S. van (2005), Cooperation in Network and Scheduling, PhD Thesis, CentER, Tilburg University, The Netherlands. 